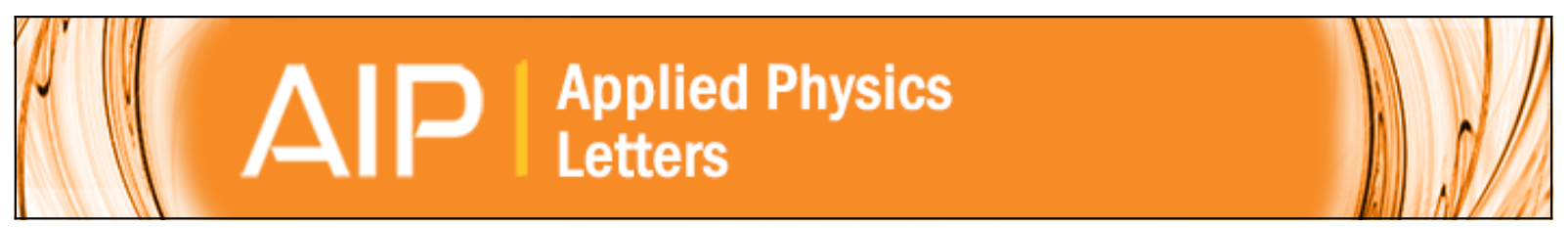

\title{
Strain engineering in monolayer WS2, MoS2, and the WS2/MoS2 heterostructure
}

Xin He, Hai Li, Zhiyong Zhu, Zhenyu Dai, Yang Yang, Peng Yang, Qiang Zhang, Peng Li, Udo Schwingenschlogl , and Xixiang Zhang

Citation: Applied Physics Letters 109, 173105 (2016); doi: 10.1063/1.4966218

View online: http://dx.doi.org/10.1063/1.4966218

View Table of Contents: http://scitation.aip.org/content/aip/journal/apl/109/17?ver=pdfcov

Published by the AIP Publishing

\section{Articles you may be interested in}

Well separated trion and neutral excitons on superacid treated MoS2 monolayers

Appl. Phys. Lett. 108, 251106 (2016); 10.1063/1.4954837

Materials properties of out-of-plane heterostructures of MoS2-WSe2 and WS2-MoSe2

Appl. Phys. Lett. 108, 063105 (2016); 10.1063/1.4941755

Strictly monolayer large continuous MoS2 films on diverse substrates and their luminescence properties Appl. Phys. Lett. 108, 042101 (2016); 10.1063/1.4940751

Phonon induced luminescence decay in monolayer MoS2 on SiO2/Si substrates

Appl. Phys. Lett. 107, 242103 (2015); 10.1063/1.4938141

A first-principles study on the effect of biaxial strain on the ultimate performance of monolayer MoS2-based double gate field effect transistor

J. Appl. Phys. 113, 163708 (2013); 10.1063/1.4803032

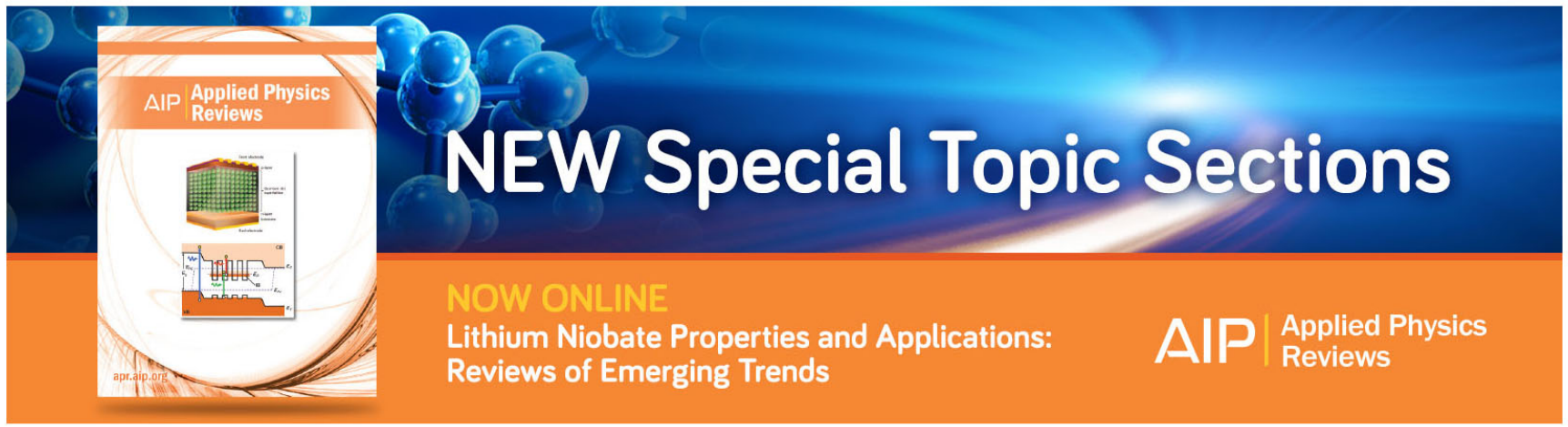




\title{
Strain engineering in monolayer $\mathrm{WS}_{2}, \mathrm{MoS}_{2}$, and the $\mathrm{WS}_{2} / \mathrm{MoS}_{2}$ heterostructure
}

\author{
Xin $\mathrm{He},{ }^{1, a)}$ Hai $\mathrm{Li},{ }^{1,2, a)}$ Zhiyong Zhu, ${ }^{3}$ Zhenyu Dai, ${ }^{1}$ Yang Yang, ${ }^{4}$ Peng Yang, ${ }^{1,2}$ \\ Qiang Zhang, ${ }^{1}$ Peng Li, ${ }^{1}$ Udo Schwingenschlogl, ${ }^{1}$ and Xixiang Zhang ${ }^{1, b)}$ \\ ${ }^{1}$ King Abdullah University of Science and Technology (KAUST), Physical Science and Engineering Division (PSE), \\ Thuwal 23955-6900, Saudi Arabia \\ ${ }^{2}$ Key Laboratory of Flexible Electronics (KLOFE) and Institute of Advanced Materials (IAM), \\ Jiangsu National Synergetic Innovation Center for Advanced Materials (SICAM), Nanjing Tech University \\ (NanjingTech), 30 South Puzhu Road, Nanjing 211816, China \\ ${ }^{3}$ KAUST Supercomputing Laboratory, King Abdullah University of Science and Technology (KAUST), \\ Thuwal 23955-6900, Saudi Arabia \\ ${ }^{4}$ Beijing National Laboratory for Condensed Matter Physics, Institute of Physics, Chinese Academy \\ of Sciences, Beijing 100190, China
}

(Received 8 August 2016; accepted 9 October 2016; published online 27 October 2016)

\begin{abstract}
Mechanically exfoliated monolayers of $\mathrm{WS}_{2}, \mathrm{MoS}_{2}$ and their van der Waals heterostructure were fabricated on flexible substrate so that uniaxial tensile strain can be applied to the twodimensional samples. The modification of the band structure under strain was investigated by micro-photoluminescence spectroscopy at room temperature as well as by first-principles calculations. Exciton and trion emissions were observed in both $\mathrm{WS}_{2}$ and the heterostructure at room temperature, and were redshifted by strain, indicating potential for applications in flexible electronics and optoelectronics. Published by AIP Publishing. [http://dx.doi.org/10.1063/1.4966218]
\end{abstract}

Graphene has attracted a huge amount of attention owing to its unique properties that are of fundamental interest and have potential applications in electronics, ${ }^{1}$ optoelectronics, ${ }^{2}$ spintronics, ${ }^{3}$ and other fields, such as sensor and energy storage. ${ }^{4,5}$ However, applications in both electronics and optoelectronics are largely limited by its zero bandgap. Tremendous efforts were made to open a bandgap in graphene, but the progress remains slow. ${ }^{6-10}$ Transition metal dichalcogenides (TMDs), being also layered materials with an S-M-S $(\mathrm{M}=\mathrm{Mo}, \mathrm{W})$ sandwich structure bonded by van der Waals forces, recently were investigated extensively as a class of alternative two-dimensional materials. ${ }^{11,12}$ This is largely due to the fact that monolayer TMDs have direct bandgaps corresponding to the region of visible light, which indicates the potential for applications in optoelectronic devices. ${ }^{13}$ Moreover, monolayer TMDs are able to withstand large strain, ${ }^{14}$ and various research groups have predicted and confirmed that the band structure can be tuned effectively by strain. ${ }^{15-21}$

van der Waals heterostructures consisting of different TMDs inherit superior electronic and optical properties from the individual TMD layers, ${ }^{13,22-26}$ and also often exhibit advanced features and functionalities not present in any component. ${ }^{27-31}$ Since many physical properties (e.g., electrical and optical) depend on the band structure, band structure tuning is of future importance. Even though for graphene and monolayer TMDs, such tuning through the application of strain was investigated extensively both theoretically and experimentally, ${ }^{14-21,32-37}$ there are only few theoretical studies on strain engineering in heterostructures, ${ }^{38-40}$ and experimental insight is still yet to be provided.

We therefore have studied the band structures of monolayers $\mathrm{WS}_{2}, \mathrm{MoS}_{2}$, and their heterostructure (WMoH) by

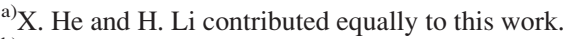

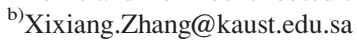

photoluminescence (PL) spectroscopy under uniaxial tensile strain from $0 \%$ to $0.64 \%$. Only a neutral direct gap exciton peak was obtained for monolayer $\mathrm{MoS}_{2}$, which showed a linear redshift under strain with a rate of $56 \mathrm{meV} / \%$ strain. Interestingly, both a neutral exciton peak (A) and a charged exciton (trion) peak (T) were observed in the PL spectra of the monolayer $\mathrm{WS}_{2}$ and the heterostructure $\mathrm{WMoH}$. Peaks A and $\mathrm{T}$ were redshifted under strain with rates of $46 \mathrm{meV} /$ $\%$ strain and $43 \mathrm{meV} / \%$ strain in monolayer $\mathrm{WS}_{2}$ and with rates of $53 \mathrm{meV} / \%$ strain and $62 \mathrm{meV} / \%$ strain in the WMoH. The shifts of the PL peaks reflect the changes of the optical bandgap as the exciton binding energy does not depend on strain. ${ }^{15}$ The bandgaps of monolayers $\mathrm{WS}_{2}, \mathrm{MoS}_{2}$ and the $\mathrm{WMoH}$, thus, can be tuned by uniaxial strain.

Monolayer samples were obtained by mechanical exfoliation from bulk $\mathrm{WS}_{2}$ and $\mathrm{MoS}_{2}$ crystals that were purchased from the HQ Graphene Company. The thin samples were exfoliated onto different $\mathrm{SiO}_{2} / \mathrm{Si}(90 \mathrm{~nm}, 500 \mu \mathrm{m})$ substrates, and the monolayer samples were identified by optical and atomic force microscopy, as shown in Figures 1(a) and 1(b) and their insets, respectively. Subsequently, the monolayer $\mathrm{WS}_{2}$ was transferred onto the monolayer $\mathrm{MoS}_{2}$ to form a double-layer heterostructure on the $\mathrm{SiO}_{2} / \mathrm{Si}$ substrate with the help of PMMA and poly(dimethylsiloxane) (PDMS) (Figure 1(c)), using the method of Li et al. ${ }^{41}$ Since the two monolayers were partially stacked, it was possible to obtain their behaviors and that of the $\mathrm{WMoH}$ simultaneously as the applied strain was varied. To improve the interlayer coupling, the $\mathrm{WMoH}$ was annealed in a mild vacuum for $12 \mathrm{~h}$ $\left(\sim 60 \mathrm{mTorr}, 120^{\circ} \mathrm{C}\right)^{42}$ and then transferred onto a $300 \mu \mathrm{m}$ thick polyethylene terephthalate (PET) film ((Figure 1(d)). A PMMA film was employed to support the sample in the transfer process and to work as a clamp that prevents it from slipping during the straining process. Since PET is a flexible substrate, a reproducible uniaxial tensile strain can be applied 

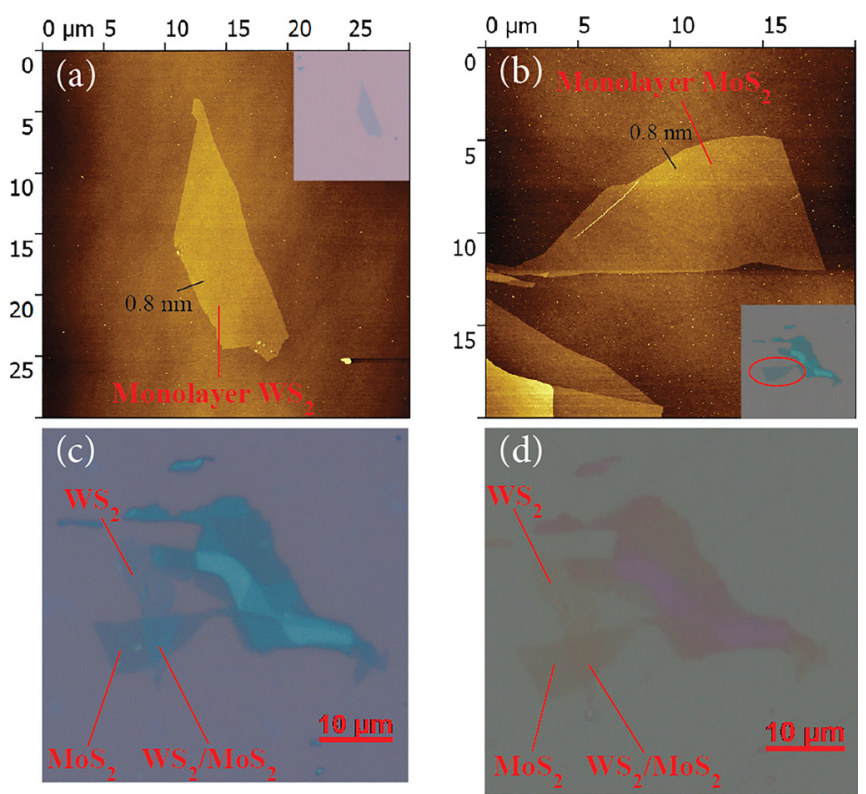

(e)

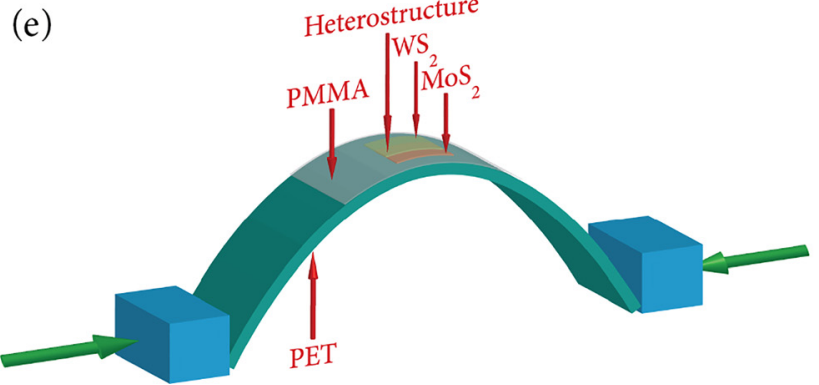

FIG. 1. (a) and (b) The AFM topographic images of monolayers $\mathrm{WS}_{2}$ and $\mathrm{MoS}_{2}$ on the $\mathrm{SiO}_{2} / \mathrm{Si}$ substrate obtained using the tapping mode; the optical images of the corresponding samples are shown in the insets. (c) and (d) The optical images of the $\mathrm{WS}_{2} / \mathrm{MoS}_{2}$ heterostructure on, respectively, the $\mathrm{SiO}_{2} /$ $\mathrm{Si}$ and PET substrates. (e) Schematic drawing of the two-point bending apparatus. The length of the PET substrate is $34.8 \mathrm{~mm}$.

to the samples using the two-point bending apparatus shown in Figure 1(e). The applied strain is given by $\varepsilon=\tau / 2 R$, where $\tau$ is the thickness and $R$ is the radius of curvature of the PET film. ${ }^{15}$ A laser $(\lambda=532 \mathrm{~nm})$ with $\sim 1 \mu \mathrm{m}^{2}$ focused spot was used for excitation. All the PL spectra were collected on a Jobin-Yvon HR 800 Raman system at room temperature. The power of the laser was kept at $\sim 5.5 \mu \mathrm{W}$ to avoid heating of the samples.

To understand how the applied strain affects the band structure, three materials are addressed: an isolated monolayer $\mathrm{WS}_{2}$, an isolated monolayer $\mathrm{MoS}_{2}$, and the $\mathrm{WMoH}$. Considering the much smaller sizes of the samples (and their relative locations) in comparison with the size of the twopoint bending apparatus (Figure 1(e)), it is safe to ignore the difference in the strain applied to them. To ensure reproducibility, each measurement was repeated three times by changing the applied strain. The dependence of the peak positions on the strain was found to be reproducible, which confirmed that there was no slip between the samples and substrate, and thus that the employed technique is reliable.

Although PL spectra of the strained monolayer $\mathrm{MoS}_{2}$ were studied by other groups, ${ }^{15,17,19}$ they were collected also in this study for comparison, see Figure S1 (supplementary material). The results are in good agreement with the previous data, ${ }^{15,17,19}$ which confirm that the technique used in this study is correct, and that there was no slip during the straining process.

Without strain, two peaks appeared in the PL spectra of $\mathrm{WS}_{2}$, see Figure 2(a). Peak A at higher energy of $2.036 \mathrm{eV}$ is due to the neutral exciton emission $\left(\mathrm{E}_{\mathrm{X}}\right)$ and peak $\mathrm{T}$ at $1.995 \mathrm{eV}$ is due to the trion emission $\left(\mathrm{E}_{\mathrm{T}}\right)$. Since the trion emission is closely related to the carrier density, it is sensitive to the laser power, as reported by Currie et al. ${ }^{43}$ The observation of trion emission under $5.5 \mu \mathrm{W}$ laser light indicated that the trion binding energy was significantly larger than the thermal energy at room temperature. The obtained binding energy of $41 \mathrm{meV}$ is calculated by subtracting $\mathrm{E}_{\mathrm{T}}$ from $E_{X}$. Under strain, both peaks redshifted, similar to the monolayer $\mathrm{MoS}_{2}$, implying a reduction in the bandgap. The positions of the exciton and trion peaks in the PL spectra are shown in Figure 2(b) as a function of the applied strain. Both peaks redshifted linearly, but with slightly different rates: $46 \mathrm{meV} / \%$ strain for peak $\mathrm{A}$ and $43 \mathrm{meV} / \%$ strain for peak $\mathrm{T}$. This result indicates clearly that the band structure can be effectively tuned by strain. ${ }^{15-17,19,34}$
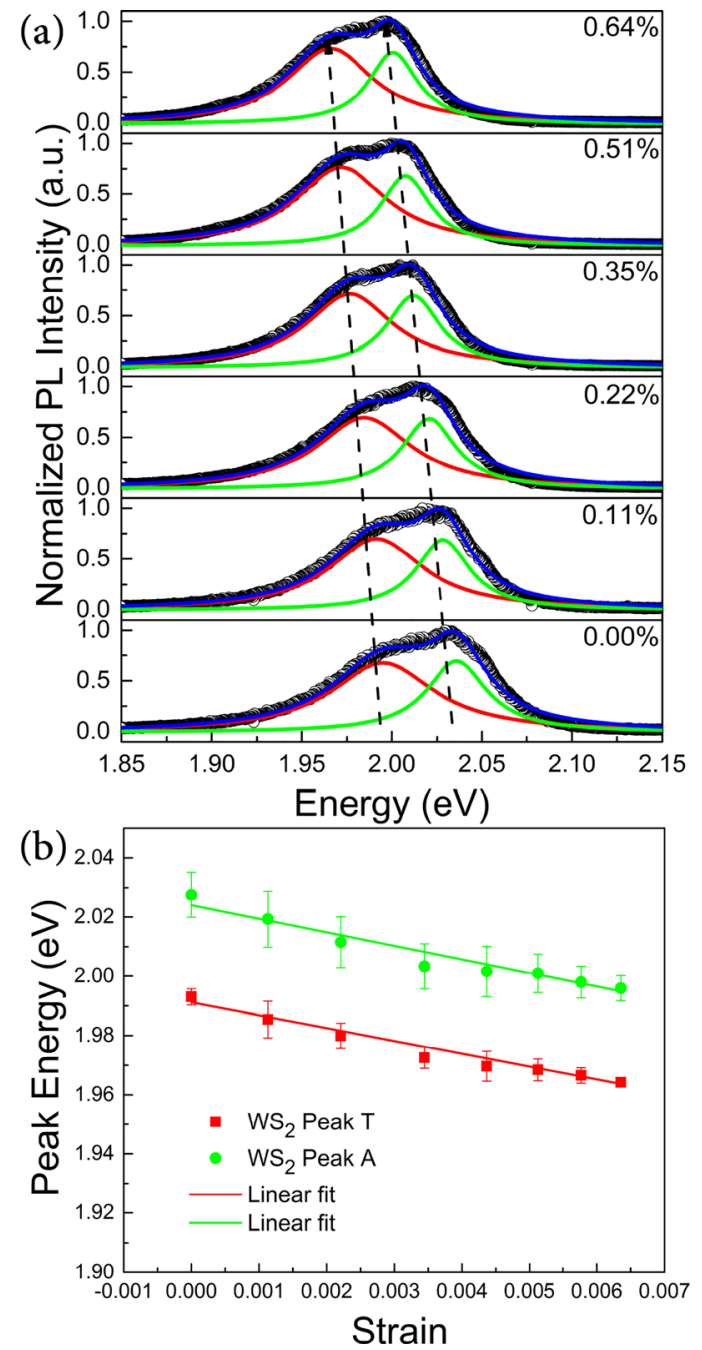

FIG. 2. (a) The PL spectra of the monolayer $\mathrm{WS}_{2}$ under strain from $0 \%$ to $0.64 \%$. Peak T (red) corresponds to the $\mathrm{WS}_{2}$ trion and peak A (green) to the $\mathrm{WS}_{2}$ exciton. The dashed lines are guides to the eye highlighting the redshift of the peaks. (b) The strain dependence of the peak T and peak A energies extracted from (a). 
The PL spectra of a typical $\mathrm{WS}_{2} / \mathrm{MoS}_{2}$ heterostructure on $\mathrm{SiO}_{2} / \mathrm{Si}$, before and after annealing, are shown in Figure S2 (supplementary material) indicating that the coupling is improved by annealing. ${ }^{42}$ The PL spectra obtained for the annealed WMoH under strain in Figure 3(a) show only the $\mathrm{WS}_{2}$ peaks. This can be explained by the fact that the annealing creates regions of strong coupling between $\mathrm{MoS}_{2}$ and $\mathrm{WS}_{2}$. In these regions, the bandgap is indirect so that the PL signal is small. In addition, their presence has effects on $\mathrm{MoS}_{2}$ in weakly coupled regions, which become depleted of holes due to the availability of the energetically higher valence band in strongly coupled regions, ${ }^{42}$ as shown in Figure 4, which suppresses the $\mathrm{MoS}_{2}$ peak. On the other hand, the $\mathrm{WS}_{2}$ peaks are not suppressed since the energy level of the conduction band minimum is not modified by the coupling. ${ }^{42}$ Analysis of the two $\mathrm{WS}_{2}$ peaks by Lorentz fitting shows that both are redshifted with increasing strain (Figure 3(b)), indicating that the energy band structure of the $\mathrm{WMoH}$ can be tuned by strain as well. The redshift rate was obtained as $53 \mathrm{meV} / \%$ strain for the exciton peak and $62 \mathrm{meV} / \%$ strain for the trion peak after linear fitting. Due to the different redshift rates, the two peaks
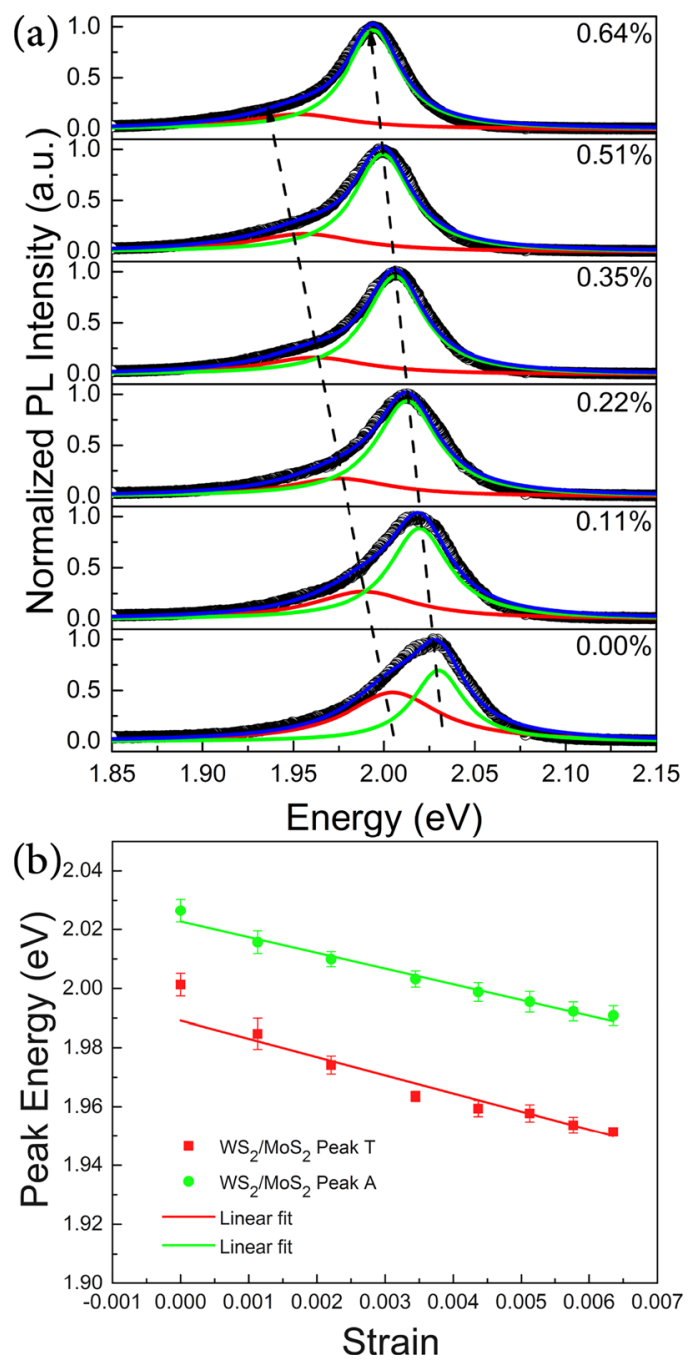

FIG. 3. (a) The PL spectra of the $\mathrm{WS}_{2} / \mathrm{MoS}_{2}$ heterostructure under strain from $0 \%$ to $0.64 \%$. Peak $\mathrm{T}$ (red) corresponds to the $\mathrm{WS}_{2}$ trion, and peak $\mathrm{A}$ (green) corresponds to the $\mathrm{WS}_{2}$ exciton. The dashed lines are guides to the eye highlighting the redshift of the peaks. (b) The strain dependence of the peak $\mathrm{T}$ and peak A energies extracted from (a).

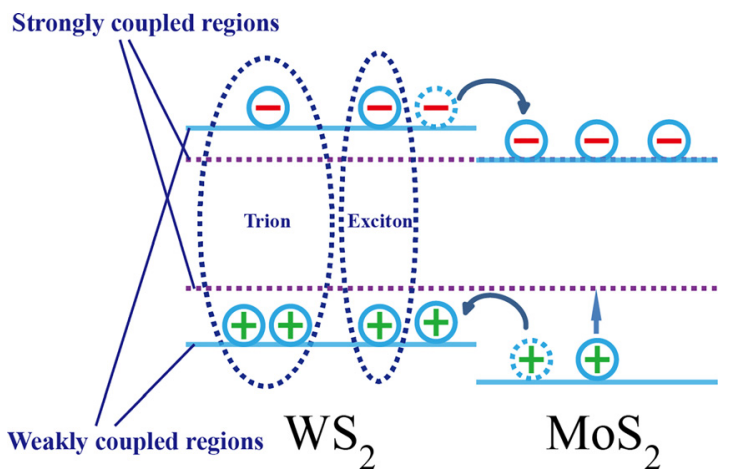

FIG. 4. The band alignment and charge transfer between the strongly and weakly coupled regions of the $\mathrm{WS}_{2} / \mathrm{MoS}_{2}$ heterostructure.

overlapped less and less, as shown in Figure 3(a). Compared with Figure 2(a), the the trion peaks are much weaker, which can be attributed to a reduced hole density in $\mathrm{WS}_{2}$ due to the presence of the strongly coupled regions as seen above.

To examine whether the strain distribution is uniform in the sample, the PL measurements were performed at three positions randomly chosen within the layers of $\mathrm{WS}_{2}$, $\mathrm{MoS}_{2}$, and $\mathrm{WMoH}$ on the PET substrate. It is found that the peak positions of the PL spectra obtained from those three positions within each layer are the same (see Figure S3) (supplementary material), indicating a uniform distribution of the applied strain. This observation is in good agreement with a previous report. ${ }^{44}$

To gain more quantitative understanding of the strain effects on the bandgap evolution, we have performed the first-principles calculations using the full-potential linearized augmented plane-wave code WIEN2k. ${ }^{45}$ The lattice constants from Ref. 46 were adopted for the monolayer $\mathrm{WS}_{2}$ $(\mathrm{a}=3.197 \AA)$ and monolayer $\mathrm{MoS}_{2}(\mathrm{a}=3.193 \AA)$, whereas for the WMoH $(\mathrm{a}=3.195 \AA)$, the average of the lattice constants of the two individual materials was used. ${ }^{46}$ Twodimensional structures were generated by adding a vacuum layer of $20 \AA$ thickness. Uniaxial strain along the zigzag or armchair direction was simulated by stretching in this direction only, in accordance with the experimental setup, as lateral movement of the sample was prohibited by the strong adhesion with the substrate. For the muffin-tin radius, values of $2.0 \mathrm{bohr}$ for $\mathrm{S}$ and $2.2 \mathrm{bohr}$ for Mo/W were used. High accuracy is achieved by employing a $10 \times 10 \times 1 \mathrm{k}$-mesh and setting $\mathrm{R}_{\mathrm{mt}} \mathrm{K}_{\max }=8.0$. The atomic positions were fully relaxed with a force tolerance of $0.1 \mathrm{mRy} / \mathrm{bohr}$ for all structures. The generalized gradient approximation to the exchange-correlation functional ${ }^{47}$ was employed for both the lattice optimization and electronic structure calculations. Spin-orbit coupling was simulated using the second variation approach.

The electronic band structures of monolayers $\mathrm{WS}_{2}$, $\mathrm{MoS}_{2}$, and the $\mathrm{WMoH}$ are addressed in Figure 5 for strain along the armchair direction and in Figure S4 for strain along the zigzag direction (supplementary material). Without strain, both the valence band maximum and conduction band minimum are located at the $\mathrm{K}$ point for $\mathrm{MoS}_{2}$ and $\mathrm{WS}_{2}$, giving rise to a direct bandgap. The size of the bandgap as a function of the applied strain is shown in Figure 6. For unstrained monolayers $\mathrm{WS}_{2}$ and $\mathrm{MoS}_{2}$, the bandgaps are $1.51 \mathrm{eV}$ and 


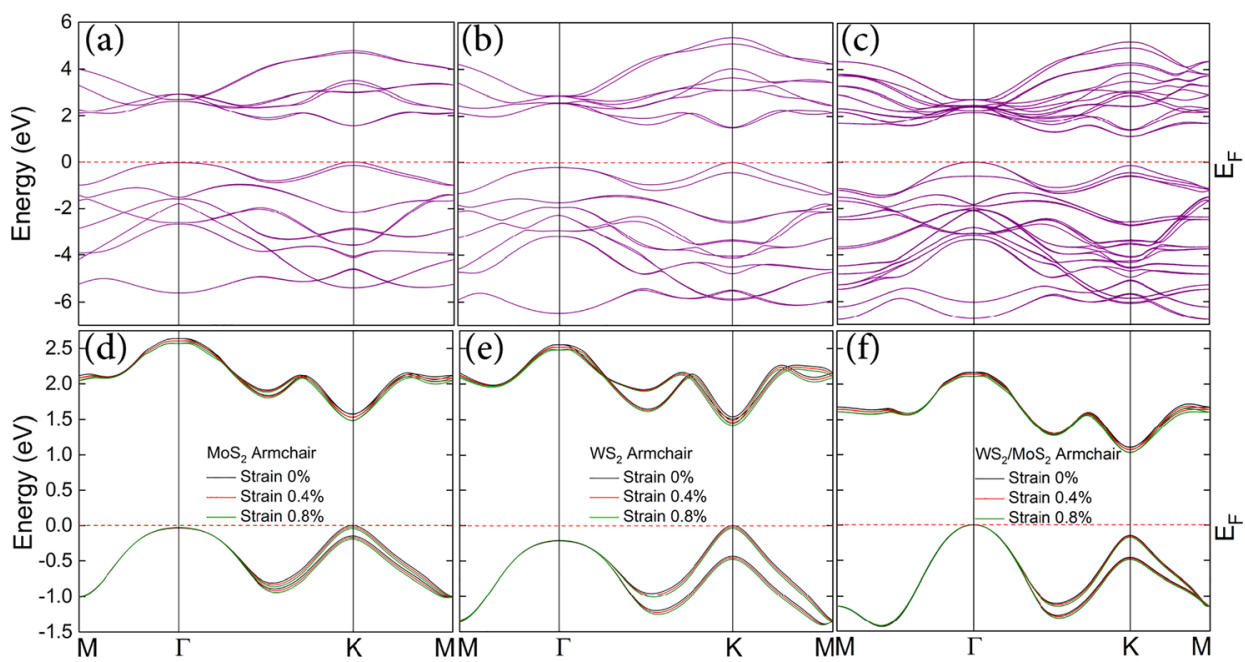

FIG. 5. The Electronic band structures of the monolayer $\mathrm{MoS}_{2}$ (a), monolayer $\mathrm{WS}_{2}(\mathrm{~b})$, and the $\mathrm{WS}_{2} / \mathrm{MoS}_{2}$ heterostructure (c) without strain. The changes of the valence band maximum and conduction band minimum are shown in (d)-(f) under strain along the armchair direction.

$1.57 \mathrm{eV}$, respectively, which are smaller than the experimental values of $2.03 \mathrm{eV}$ and $1.87 \mathrm{eV}$, since density functional theory underestimates bandgaps. While for $\mathrm{WMoH}$ without strain, the valence band maximum and conduction band minimum are located at the $\Gamma$ and $\mathrm{K}$ point, respectively, resulting in an indirect bandgap. Under strain, the bandgap is modified in a linear manner, see Figure 6, similar along the zigzag and armchair directions. For $1 \%$ strain, the predicted bandgap reductions are $64 \mathrm{meV}$ for $\mathrm{WS}_{2}, 55 \mathrm{meV}$ for $\mathrm{MoS}_{2}$, and $65 \mathrm{meV}$ for the $\mathrm{WMoH}$, which are consistent with our experiments.

We have investigated the bandgap modulation of monolayers $\mathrm{WS}_{2}, \mathrm{MoS}_{2}$, and the $\mathrm{WMoH}$ under strain, using both experimental and theoretical methods. Neutral excitons with large binding energies were observed in both the unstrained and strained samples in each case, whereas charged excitons (trions) were only observed in monolayer $\mathrm{WS}_{2}$ and the WMoH. Both the neutral exciton (56 meV/\%strain, $46 \mathrm{meV} /$ $\%$ strain, and $53 \mathrm{meV} / \%$ strain for monolayers $\mathrm{MoS}_{2}, \mathrm{WS}_{2}$, and the $\mathrm{WMoH}$, respectively) and trion (43 meV/\% strain and $62 \mathrm{meV} / \%$ strain for monolayer $\mathrm{WS}_{2}$ and the $\mathrm{WMoH}$, respectively) peaks showed redshifts under strain, indicating decreasing bandgaps. The tunability of the band structures by strain implies potential applications in flexible electronics and optoelectronics.

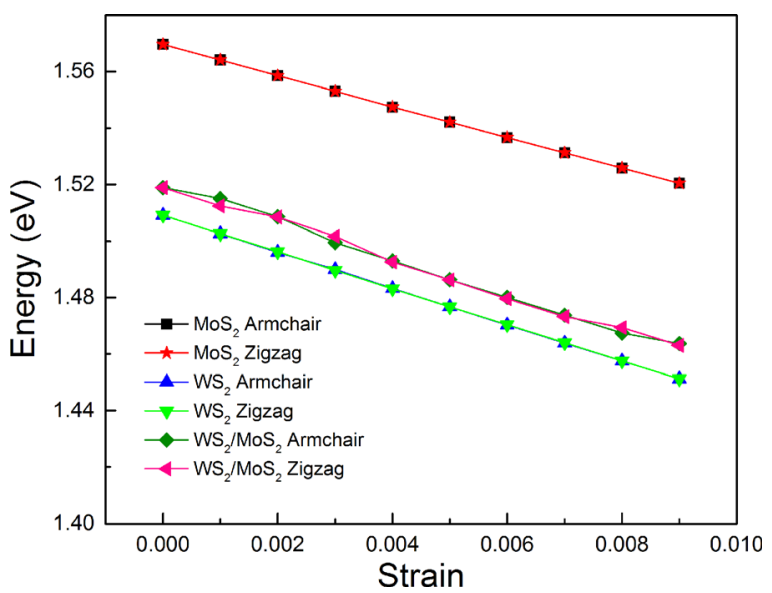

FIG. 6. Theoretical evolution of the bandgap of monolayers $\mathrm{WS}_{2}, \mathrm{MoS}_{2}$, and the $\mathrm{WS}_{2} / \mathrm{MoS}_{2}$ heterostructure under strain from $0 \%$ to $0.9 \%$ along the zigzag and armchair directions.
See supplementary material for the PL spectra of the monolayer $\mathrm{MoS}_{2}$ under strain, PL spectra of a typical $\mathrm{WS}_{2} /$ $\mathrm{MoS}_{2}$ heterostructure before and after annealing, PL spectra uniformity in $\mathrm{WS}_{2}, \mathrm{MoS}_{2}$, and $\mathrm{WS}_{2} / \mathrm{MoS}_{2}$ regions before and after straining and electronic band structures of monolayers $\mathrm{WS}_{2}, \mathrm{MoS} 2$, and the $\mathrm{WMoH}$ under strain along the zigzag direction.

The research reported in this publication was supported by funding from King Abdullah University of Science and Technology (KAUST). H. Li acknowledges financial support from the National Natural Science Foundation of China (Grant No. 21571101), Specially Appointed Professors by Universities in Jiangsu Province, and the Natural Science Foundation of the Jiangsu Higher Education Institutions of China (Grant No. 15KJB430016).

${ }^{1}$ F. Xia, D. B. Farmer, Y. M. Lin, and P. Avouris, "Graphene field-effect transistors with high on/off current ratio and large transport band gap at room temperature," Nano Lett. 10, 715-718 (2010).

${ }^{2}$ F. Bonaccorso, Z. Sun, T. Hasan, and A. C. Ferrari, "Graphene photonics and optoelectronics," Nat. Photonics 4, 611-622 (2010).

${ }^{3}$ N. Tombros, C. Jozsa, M. Popinciuc, H. T. Jonkman, and B. J. van Wees, "Electronic spin transport and spin precession in single graphene layers at room temperature," Nature 448, 571-574 (2007).

${ }^{4}$ F. Schedin, A. K. Geim, S. V. Morozov, E. W. Hill, P. Blake, M. I. Katsnelson, and K. S. Novoselov, "Detection of individual gas molecules adsorbed on graphene," Nat. Mater. 6, 652-655 (2007).

${ }^{5}$ M. D. Stoller, S. Park, Y. Zhu, J. An, and R. S. Ruoff, "Graphene-based ultracapacitors," Nano Lett. 8, 3498-3502 (2008).

${ }^{6}$ J. Bai, X. Zhong, S. Jiang, Y. Huang, and X. Duan, "Graphene nanomesh," Nat. Nanotechnol. 5, 190-194 (2010).

${ }^{7}$ A. Kazemi, X. He, S. Alaie, J. Ghasemi, N. M. Dawson, F. Cavallo, T. G. Habteyes, S. R. J. Brueck, and S. Krishna, "Large-area semiconducting graphene nanomesh tailored by interferometric lithography," Sci. Rep. 5, 11463 (2015).

${ }^{8}$ L. Liu, Y. Zhang, W. Wang, C. Gu, X. Bai, and E. Wang, "Nanosphere lithography for the fabrication of ultranarrow graphene nanoribbons and on-chip bandgap tuning of graphene," Adv. Mater. 23, 1246-1251 (2011). ${ }^{9}$ J. G. Son, M. Son, K. J. Moon, B. H. Lee, J. M. Myoung, M. S. Strano, M. H. Ham, and C. A. Ross, "Sub-10 nm graphene nanoribbon array fieldeffect transistors fabricated by block copolymer lithography," Adv. Mater. 25, 4723-4728 (2013).

${ }^{10}$ Z. Zeng, X. Huang, Z. Yin, H. Li, Y. Chen, H. Li, Q. Zhang, J. Ma, F. Boey, and H. Zhang, "Fabrication of graphene nanomesh by using an anodic aluminum oxide membrane as a template," Adv. Mater. 24, 4138-4142 (2012).

${ }^{11}$ S. Z. Butler, S. M. Hollen, L. Cao, Y. Cui, J. A. Gupta, H. R. Gutierrez, T. F. Heinz, S. S. Hong, J. Huang, A. F. Ismach, E. Johnston-Halperin, 
M. Kuno, V. V. Plashnitsa, R. D. Robinson, R. S. Ruoff, S. Salahuddin, J. Shan, L. Shi, M. G. Spencer, M. Terrones, W. Windl, and J. E. Goldberger, "Progress, challenges, and opportunities in two-dimensional materials beyond graphene," ACS Nano 7, 2898-2926 (2013).

${ }^{12}$ M. Xu, T. Liang, M. Shi, and H. Chen, "Graphene-like two-dimensional materials," Chem. Rev. 113, 3766-3798 (2013).

${ }^{13}$ Q. H. Wang, K. Kalantar-Zadeh, A. Kis, J. N. Coleman, and M. S. Strano, "Electronics and optoelectronics of two-dimensional transition metal dichalcogenides," Nat. Nanotechnol. 7, 699-712 (2012).

${ }^{14}$ A. Castellanos-Gomez, M. Poot, G. A. Steele, H. S. van der Zant, N. Agrait, and G. Rubio-Bollinger, "Mechanical properties of freely suspended semiconducting graphene-like layers based on $\mathrm{MoS}_{2}$," Nanoscale Res. Lett. 7, 233 (2012).

${ }^{15}$ H. J. Conley, B. Wang, J. I. Ziegler, R. F. Haglund, S. T. Pantelides, and K. I. Bolotin, "Bandgap engineering of strained monolayer and bilayer $\mathrm{MoS}_{2}$," Nano Lett. 13, 3626-3630 (2013).

${ }^{16}$ S. B. Desai, G. Seol, J. S. Kang, H. Fang, C. Battaglia, R. Kapadia, J. W. Ager, J. Guo, and A. Javey, "Strain-induced indirect to direct bandgap transition in multilayer WSe 2 ," Nano Lett. 14, 4592-4597 (2014).

${ }^{17}$ K. He, C. Poole, K. F. Mak, and J. Shan, "Experimental demonstration of continuous electronic structure tuning via strain in atomically thin $\mathrm{MoS}_{2}$," Nano Lett. 13, 2931-2936 (2013).

${ }^{18}$ Y. Y. Hui, X. Liu, W. Jie, N. Y. Chan, J. Hao, Y. T. Hsu, L. J. Li, W. Guo, and S. P. Lau, "Exceptional tunability of band energy in a compressively strained trilayer $\mathrm{MoS}_{2}$ sheet," ACS Nano 7, 7126-7131 (2013).

${ }^{19}$ C. R. Zhu, G. Wang, B. L. Liu, X. Marie, X. F. Qiao, X. Zhang, X. X. Wu, H. Fan, P. H. Tan, T. Amand, and B. Urbaszek, "Strain tuning of optical emission energy and polarization in monolayer and bilayer $\mathrm{MoS}_{2}$," Phys. Rev. B 88, 121301 (2013).

${ }^{20}$ H. Peelaers and C. G. Van de Walle, "Effects of strain on band structure and effective masses in $\mathrm{MoS}_{2}$," Phys. Rev. B 86, 241401 (2012).

${ }^{21}$ H. Shi, H. Pan, Y. W. Zhang, and B. I. Yakobson, "Quasiparticle band structures and optical properties of strained monolayer $\mathrm{MoS}_{2}$ and $\mathrm{WS}_{2}$," Phys. Rev. B 87, 155304 (2013).

${ }^{22}$ K. F. Mak, K. He, C. Lee, G. H. Lee, J. Hone, T. F. Heinz, and J. Shan, "Tightly bound trions in monolayer $\mathrm{MoS}_{2}$," Nat. Mater. 12, 207-211 (2013).

${ }^{23}$ K. F. Mak, K. L. McGill, J. Park, and P. L. McEuen, "The valley Hall effect in $\mathrm{MoS}_{2}$ transistors," Science 344, 1489-1492 (2014).

${ }^{24}$ B. Radisavljevic, A. Radenovic, J. Brivio, V. Giacometti, and A. Kis, "Single-layer $\mathrm{MoS}_{2}$ transistors," Nat. Nanotechnol. 6, 147-150 (2011).

${ }^{25}$ W. Wu, L. Wang, Y. Li, F. Zhang, L. Lin, S. Niu, D. Chenet, X. Zhang, Y. Hao, T. F. Heinz, J. Hone, and Z. L. Wang, "Piezoelectricity of singleatomic-layer $\mathrm{MoS}_{2}$ for energy conversion and piezotronics," Nature 514, 470-474 (2014)

${ }^{26}$ Y. J. Zhang, T. Oka, R. Suzuki, J. T. Ye, and Y. Iwasa, "Electrically switchable chiral light-emitting transistor," Science 344, 725-728 (2014).

${ }^{27}$ X. Hong, J. Kim, S. F. Shi, Y. Zhang, C. Jin, Y. Sun, S. Tongay, J. Wu, Y. Zhang, and F. Wang, "Ultrafast charge transfer in atomically thin $\mathrm{MoS}_{2} /$ $\mathrm{WS}_{2}$ heterostructures," Nat. Nanotechnol. 9, 682-686 (2014).

${ }^{28}$ C. H. Lee, G. H. Lee, A. M. van der Zande, W. Chen, Y. Li, M. Han, X. Cui, G. Arefe, C. Nuckolls, T. F. Heinz, J. Guo, J. Hone, and P. Kim, "Atomically thin p-n junctions with van der Waals heterointerfaces," Nat. Nanotechnol. 9, 676-681 (2014).

${ }^{29}$ P. Rivera, J. R. Schaibley, A. M. Jones, J. S. Ross, S. Wu, G. Aivazian, P. Klement, K. Seyler, G. Clark, N. J. Ghimire, J. Yan, D. G. Mandrus, W. Yao, and $\mathrm{X}$. Xu, "Observation of long-lived interlayer excitons in monolayer $\mathrm{MoSe}_{2}-\mathrm{WSe}_{2}$ heterostructures," Nat. Commun. 6, 6242 (2015).

${ }^{30}$ F. Withers, O. Del Pozo-Zamudio, A. Mishchenko, A. P. Rooney, A. Gholinia, K. Watanabe, T. Taniguchi, S. J. Haigh, A. K. Geim, A. I.
Tartakovskii, and K. S. Novoselov, "Light-emitting diodes by bandstructure engineering in van der Waals heterostructures," Nat. Mater. 14, 301-306 (2015)

${ }^{31}$ W. J. Yu, Y. Liu, H. Zhou, A. Yin, Z. Li, Y. Huang, and X. Duan, "Highly efficient gate-tunable photocurrent generation in vertical heterostructures of layered materials," Nat. Nanotechnol. 8, 952-958 (2013).

${ }^{32}$ C. Rice, R. J. Young, R. Zan, U. Bangert, D. Wolverson, T. Georgiou, R. Jalil, and K. S. Novoselov, "Raman-scattering measurements and firstprinciples calculations of strain-induced phonon shifts in monolayer $\mathrm{MoS}_{2}$," Phys. Rev. B 87, 081307 (2013).

${ }^{33}$ Y. Wang, C. Cong, C. Qiu, and T. Yu, "Raman spectroscopy study of lattice vibration and crystallographic orientation of monolayer $\mathrm{MoS}_{2}$ under uniaxial strain," Small 9, 2857-2861 (2013).

${ }^{34}$ Y. Wang, C. Cong, W. Yang, J. Shang, N. Peimyoo, Y. Chen, J. Kang, J. Wang, W. Huang, and T. Yu, "Strain-induced direct-indirect bandgap transition and phonon modulation in monolayer $\mathrm{WS}_{2}$," Nano Res. 8, 2562-2572 (2015).

${ }^{35}$ X. He, L. Gao, N. Tang, J. Duan, F. Mei, H. Meng, F. Lu, F. Xu, X. Wang, X. Yang, W. Ge, and B. Shen, "Electronic properties of polycrystalline graphene under large local strain,” Appl. Phys. Lett. 104, 243108 (2014).

${ }^{36}$ X. He, L. Gao, N. Tang, J. Duan, F. Xu, X. Wang, X. Yang, W. Ge, and B. Shen, "Shear strain induced modulation to the transport properties of graphene," Appl. Phys. Lett. 105, 083108 (2014).

${ }^{37}$ X. He, N. Tang, X. Sun, L. Gan, F. Ke, T. Wang, F. Xu, X. Wang, X. Yang, W. Ge, and B. Shen, "Tuning the graphene work function by uniaxial strain,” Appl. Phys. Lett. 106, 043106 (2015).

${ }^{38}$ N. Lu, H. Guo, L. Li, J. Dai, L. Wang, W. N. Mei, X. Wu, and X. C. Zeng, " $\mathrm{MoS}_{2} / \mathrm{MX}_{2}$ heterobilayers: Bandgap engineering via tensile strain or external electrical field," Nanoscale 6, 2879-2886 (2014).

${ }^{39}$ L. Huang, Y. Li, Z. Wei, and J. Li, "Strain induced piezoelectric effect in black phosphorus and $\mathrm{MoS}_{2}$ van der Waals heterostructure," Sci. Rep. 5, 16448 (2015).

${ }^{40}$ H. Kumar, D. Er, L. Dong, J. Li, and V. B. Shenoy, "Elastic deformations in 2D van der Waals heterostructures and their impact on optoelectronic properties: Predictions from a multiscale computational approach," Sci. Rep. 5, 10872 (2015)

${ }^{41}$ H. Li, J. Wu, X. Huang, Z. Yin, J. Liu, and H. Zhang, "A universal, rapid method for clean transfer of nanostructures onto various substrates," ACS Nano 8, 6563-6570 (2014).

${ }^{42}$ S. Tongay, W. Fan, J. Kang, J. Park, U. Koldemir, J. Suh, D. S. Narang, K. Liu, J. Ji, J. Li, R. Sinclair, and J. Wu, "Tuning interlayer coupling in large-area heterostructures with CVD-grown $\mathrm{MoS}_{2}$ and $\mathrm{WS}_{2}$ monolayers," Nano Lett. 14, 3185-3190 (2014).

${ }^{43}$ M. Currie, A. T. Hanbicki, G. Kioseoglou, and B. T. Jonker, "Optical control of charged exciton states in tungsten disulfide," Appl. Phys. Lett. 106, 201907 (2015).

${ }^{44}$ R. J. Young, L. Gong, I. A. Kinloch, I. Riaz, R. Jalil, and K. S. Novoselov, "Strain mapping in a graphene monolayer nanocomposite," ACS Nano 5, 3079-3084 (2011).

${ }^{45}$ P. Blaha, K. Schwarz, G. K. H. Madsen, D. Kvasnicka, and L. Luitz, WIEN2k, An Augmented Plane Wave + Local Orbitals Program for Calculating Crystal Properties (Karlheinz Schwarz, Techn. Universität Wien, Austria, 2001), ISBN: 3-9501031-1-2.

${ }^{46}$ Z. Zhu, Y. Cheng, and U. Schwingenschlogl, "Giant spin-orbit-induced spin splitting in two-dimensional transition-metal dichalcogenide semiconductors," Phys. Rev. B 84, 153402 (2011).

${ }^{47}$ J. P. Perdew, K. Burke, and M. Ernzerhof, "Generalized gradient approximation made simple," Phys. Rev. Lett. 77, 3865-3868 (1996). 